\title{
Perfil espacial e demográfico da Hanseníase no norte do país, no período de 2014 a 2019
}

Spatial and demographic profile of Hansen's disease in the north of the country, from 2014 to 2019

Perfil espacial y demográfico de la Lepra en el norte del país, de 2014 a 2019

Débora de Jesus Sizo Oliveira ${ }^{1 *}$, Adrielle Barbosa Palmeira ${ }^{1}$, Elem Cristina Rodrigues Chaves ${ }^{1}$, Brendol Phelipe Silva dos Santos ${ }^{1}$, Kleber das Neves Trindade Júnior ${ }^{1}$, Beatriz Fernanda Fernandes de Andrade ${ }^{1}$, Sâmia Carolina Costa Ferreira ${ }^{1}$, Wailla Novaes do Rosário ${ }^{1}$, Victória Gabriella Dias de Sousa', Maria Helena Rodrigues de Mendonça'.

\section{RESUMO}

Objetivo: Traçar distribuição espacial e demográfica da Hanseníase na região Norte do Brasil, no período de 2014-2019. Métodos: Estudo transversal, descritivo e quali-quantitativa da distribuição da doença. Resultado: Foram registrados 32.618 casos de hanseníase na Região Norte, com maior frequência no Pará $(50,8 \%)$, em indivíduos masculinos $(59 \%)$, na faixa de $30-50$ anos $(53,5 \%)$ e baixa escolaridade $(46,2 \%)$. Referente ao esgotamento sanitário, $85,7 \%$ dos estados do Norte estão abaixo do esperado (8,02\%-33,91\%) e, considerando os municípios atendidos, Amazonas (6,5\%), Pará $(9 \%)$ e Acre $(9,1 \%)$ expõem menores resultados. Isoladamente aos municípios do Pará, considerando cidades com maiores taxas de incidência, destacam-se Tailândia $(64,2 \%)$, Altamira $(61,6 \%)$ e Parauapebas $(60 \%)$, sendo Tailândia com menor cobertura de equipes Saúde da Família (28,4\%); e, menor incidência, Santarém (15,1\%), Bragança (15,9\%) e Belém (17,6\%). Conclusão: Boa cobertura da eSF resulta no controle da transmissão da doença, logo, a importância do desenvolvimento de políticas de saúde para estados e municípios com maior vulnerabilidade, visando a melhor assistência em saúde.

Palavras-chave: Hanseníase, Epidemiologia, Determinantes sociais de saúde.

\section{ABSTRACT}

Objective: To trace the spatial and demographic distribution of Leprosy in the Northern region of Brazil, in the period 2014-2019. Methods: Cross-sectional, descriptive and quali-quantitative study of disease distribution. Result: There were 32,618 cases of leprosy in the Northern Region, with higher frequency in Pará (50.8\%), in males (59\%), in the 30-50 years age group (53.5\%) and low schooling (46.2\%). Regarding sanitary sewage, $85.7 \%$ of the Northern states are below expected (8.02\%-33.91\%) and, considering the municipalities served, Amazonas (6.5\%), Pará $(9 \%)$ and Acre $(9.1 \%)$ expose lower results. In isolation, the municipalities of Pará, considering cities with higher incidence rates, Thailand (64.2\%), Altamira $(61.6 \%)$ and Parauapebas $(60 \%)$, thailand being with the lowest coverage of Family Health teams $(28.4 \%)$; and, lower incidence, Santarém (15.1\%), Bragança (15.9\%) and Belém (17.6\%). Conclusion: Good coverage of the eSF results in the control of the transmission of the disease, therefore, the importance of developing health policies for states and municipalities with greater vulnerability, aiming at better health care.

Key words: Hansen's disease, Epidemiology, Social determinants of health.

${ }^{1}$ Centro Universitário (FIBRA), Belém - PA. `E-mail: deborahsizo37@gmail.com

SUBMETIDO EM: 3/2021

ACEITO EM: 4/2021

PUBLICADO EM: 4/2021 


\section{RESUMEN}

Objetivo: Rastrear distribución espacial y demográfica de lepra en la región norte de Brasil, en el período 2014-2019. Métodos: Estudio transversal, descriptivo y cuantificativo de la distribución de la enfermedad. Resultado: Hubo 32.618 casos de lepra la Región Norte, con mayor frecuencia en Pará (50,8\%), en hombres (59\%), en el grupo de edad de 30 a 50 años $(53,5 \%)$ y la escolarización baja $(46,2 \%)$. En cuanto a las aguas residuales sanitarias, el $85,7 \%$ de los estados del norte están por debajo de lo esperado $(8,02 \%-33,91 \%)$ y, teniendo en cuenta los municipios atendidos, Amazonas (6,5\%), Pará $(9 \%)$ y Acre $(9,1 \%)$ exponer resultados más bajos. De forma aislada, los municipios de Pará, considerando ciudades con mayores tasas de incidencia, Tailandia $(64,2 \%)$, Altamira $(61,6 \%)$ y Parauapebas $(60 \%)$, Tailandia con la menor cobertura de equipos de Salud Familiar (28,4\%); y, menor incidencia, Santarém (15,1\%), Bragança (15,9\%) y Belén (17,6\%). Conclusión: Buena cobertura del eSF se traduce en el control de la transmisión de la enfermedad, por lo tanto, la importancia de desarrollar políticas de salud dirigidas a los estados y municipios con mayor vulnerabilidad, con el objetivo de mejorar la atención de la salud.

Palabras clave: Lepra, Epidemiología, Los determinantes sociales de la salud.

\section{INTRODUÇÃO}

A Hanseníase é uma doença infectocontagiosa, de notificação compulsória que integra o grupo de doenças tropicais negligenciadas e foi durante muito tempo uma doença apontada pela discriminação e exclusão do doente. A falta de informações sobre o seu modo de transmissão, controle, cura e o medo da exclusão social, contribuíram, certamente, para que a hanseníase se tornasse uma doença temida, fazendo com que os doentes fossem segregados da população (BASEGGIO RC, 2016; NASCIMENTO DS, et al, 2020).

O mal de Hansen, como também é conhecido, se trata de uma doença que apresenta uma evolução prolongada, causada por uma bactéria gram-positiva chamada Mycobacterium leprae, considerada ÁlcoolÁcido Resistente (BAAR). O bacilo se aloja nas células de Schwann, acometendo principalmente os nervos periféricos e pele, causando lesões nas mãos, pés e olhos, resultando em lesões de natureza combinada dermatoneurológicas (MENDONÇA VA, et al., 2008; SOUZA CDF, et al., 2020).

A transmissão ocorre de pessoa a pessoa pelas vias respiratórias, através do convívio de indivíduos susceptíveis com doentes sem tratamento adequado, por isso, possui uma alta infectividade, sendo capaz de afetar muitos indivíduos, no entanto sua patogenicidade é baixa, portanto poucos adoecem (GONÇALVES NV, et al., 2018; SOUZA CDF, et al., 2020). Essas propriedades dependem de características específicas do bacilo, de sua relação com o hospedeiro, além de condições individuais, como determinantes sociais e o grau de endemicidade do meio, que também estão relacionadas ao processo de transmissão ativa (BRASIL, 2001).

De acordo com a Organização Mundial da Saúde (OMS), o Brasil foi classificado como um país de alta carga para a hanseníase, ocupando o segundo lugar na relação de países com maior número de casos no mundo, sendo assim a hanseníase ainda se apresenta como um sério problema de Saúde Pública a ser equacionado. A vista disso, foi implementado políticas públicas por intermédio do Plano Nacional de Eliminação da Hanseníase, a fim de promover uma maior Atenção Primária à Saúde (APS) e uma melhor qualidade nos serviços de saúde, para o controle da doença. Desta forma, o enfrentamento da Hanseníase é prioridade para o Ministério da Saúde, sendo as principais estratégias de ação a detecção precoce de casos e o exame de contatos, com o intuito de prevenir as incapacidades físicas e possibilitar a quebra da cadeia de transmissão (RIBEIRO MDA, et al., 2018; BRASIL, 2020).

O contexto epidemiológico da doença no Brasil é considerado não homogêneo, devido à grande variação de casos em diferentes escalas geográficas, apresentando uma baixa prevalência na região Sul, enquanto no Centro-oeste, Norte e Nordeste, a relevância da endemia mostra estar distante de ser vencida. Todas estas regiões apresentam características históricas, socioeconômicas, demográficas e de políticas públicas, nas quais se destacam as condições de vida desfavoráveis das populações humanas e as dificuldades de 
acesso aos serviços de saúde, fatores esses que estão relacionados com os determinantes sociais, fazendo com que, os níveis endêmicos e hiperendêmicos sejam atingidos em vários estados (CAVALCANTI CDTV e SILVA CGC, 2008; JUNIOR FACP, 2011).

Partindo desse princípio, um dos principais aspectos que a saúde coletiva busca identificar é a relação das variáveis socioeconômicas e epidemiológicas, por meio do Índice de Condições de Vida (ICV), da cobertura da Estratégia de Saúde da Família (ESF), que visa à reorganização da atenção básica no País, com o auxílio de uma equipe multiprofissional, seguindo os preceitos do Sistema Único de Saúde, e da taxa de detecção anual de casos novos da hanseníase (TDMH) com a ocorrência da doença (BASEGGIO RC, 2016; GONÇALVES NV, et al., 2018).

Através de um olhar holístico para a epidemiologia social é possível compreender melhor a relação desses condicionantes e determinantes sociais com a doença, visto que a hanseníase é mais incidente em locais com baixa qualidade de vida, no qual tem apresentado um alto nível de endemicidade, evidenciando a necessidade de intensificação de ações de vigilância epidemiológica, a fim de aumentar a detecção precoce de casos novos e contribuir para o alcance das metas de controle da hanseníase. Portanto, esta pesquisa é de grande relevância e importância, em virtude da alta prevalência da doença na Região Norte, quando comparada ao restante do Brasil (GONÇALVES NV, et al., 2018).

Desse modo, o presente estudo teve como objetivo traçar o perfil epidemiológico da Hanseníase e a distribuição espacial e demográfica da região Norte do Brasil, no período de 2014 a 2019.

\section{MÉTODOS}

Trata-se de um estudo epidemiológico, transversal e descritivo com uma abordagem quali-quantitativa, com o intuito de analisar a distribuição da doença em função do tempo e região de estudo e os possíveis determinantes que influenciam em sua propagação.

As variáveis independentes foram selecionadas a partir de uma revisão de literatura ampla e especializada, para identificar os indicadores epidemiológicos e os fatores sociodemográficos associados à ocorrência da hanseníase, sendo estabelecidos: a taxa de incidência e percentagem dos casos, o sexo, a faixa etária e a escolaridade, obtidos por meio do Departamento de Doenças Crônicas e Infecções Sexualmente Transmissíveis (DCCl). Para obtenção do número e cobertura de equipes de Saúde da Família foi utilizado a Sala de Apoio à Gestão Estratégica (SAGE), de acordo com as Unidades Federativas da região Norte do Brasil e os municípios do Estado do Pará.

Para os coeficientes relacionados ao saneamento básico foi utilizado o Sistema Nacional de Informação sobre Saneamento (SNIS), no qual analisa os indicadores operacionais e os investimentos dos serviços de água e esgoto, sendo de interesse para o estudo: o índice de atendimento total de água; o índice de coleta de esgoto; a quantidade de municípios atendidos com abastecimento de água e esgotamento sanitário, e os que apresentam a delegação em vigor ou vencida. Ademais, também se utilizou o Índice de Desenvolvimento Humano (IDH/IDHM), através do Instituto Brasileiro de Geografia e Estatística (IBGE), como indicador de renda, saúde e educação regional e/ou municipal.

O estudo contemplou o período de 2014 a 2019, para a obtenção dos dados de forma concreta e precisa, de acordo com os Sistemas de Informação e os boletins epidemiológicos já existentes. A partir desta coleta, foi efetuado o registro das informações por meio da criação de planilhas e tabelas na plataforma Microsoft Excel 2013 e posteriormente, a produção dos gráficos e tabelas resultantes.

Os dados coletados concernentes a taxa de detecção de casos da Hanseníase, se refere à incidência. Desta forma, foi necessário aplicar a seguinte fórmula: taxa da incidência x população atingida/100.000, para a obtenção do valor absoluto de casos que posteriormente foram transformados para percentuais.

As pesquisas foram realizadas através das plataformas Google Acadêmico, Scientific Electronic Library Online (SciELO), Biblioteca Virtual em Saúde (BVS) e os boletins epidemiológicos disponibilizados pelo Ministério da Saúde (MS), a fim de reunir estudos relevantes para o tema em questão. Foram utilizados, para 
busca dos artigos, os seguintes descritores: Hanseníase; Epidemiologia; Determinantes Sociais de Saúde, que também validaram os critérios de inclusão do estudo. Os critérios de inclusão definidos para a seleção dos artigos foram: Artigos preferencialmente em português, que retratassem a temática em questão e os fatores que estão relacionados, de forma plena ou parcial, conforme o objeto de estudo.

Figura 1 - Fluxograma de integração metodológica contínua para busca, registro e processamento de dados.

Estudo epidemiológico, transversal e descritivo com uma abordagem quali-quantitativa
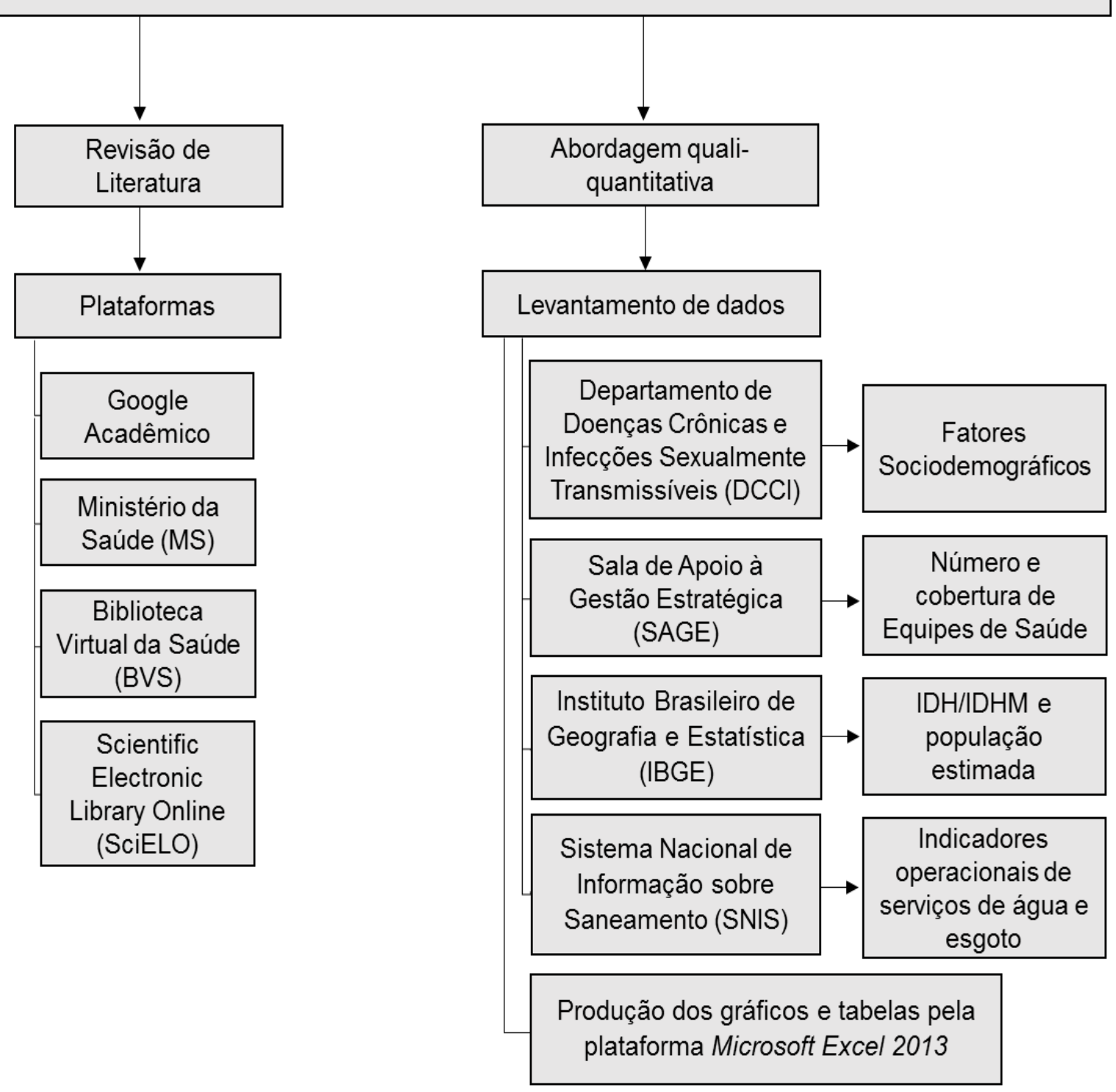

Fonte: Oliveira DJS, et al., 2021.

\section{RESULTADOS E DISCUSSÃO}

De acordo com os dados obtidos no Departamento de Doenças de Condições Crônicas e Infecções Sexualmente Transmissíveis (DCCl) na Região Norte, no período de 2014 a 2019, foram registrados 32.618 casos novos de Hanseníase, com maior concentração no estado do Pará $(50,8 \%)$, seguido de Tocantins (24\%), Rondônia $(10,6 \%)$, Amazonas $(8,6 \%)$, Acre $(2,3 \%)$, Amapá $(2 \%)$ e Roraima $(1,7 \%)$, os quais apresentaram significativas variações na distribuição da doença em função do tempo. Ao analisar os dados, foi observada estabilidade na incidência de casos em 2014 a 2017, variando entre 35,5 a 28,8, todavia, de 2017 a 2018, os casos aumentam cerca de 12,3\%, chegando a incidência de 31,9, com distribuição de casos 
no Tocantins (22,1\%), Rondônia (21,2\%), Roraima (18,7\%), Acre (17,6\%), Amapá (16,7\%), Pará (15,5\%) e Amazonas (15,1\%) (Figura 1).

Figura 1 - Demonstra a percentagem e incidência de casos de hanseníase nas Unidades Federativas da região Norte, no período de 2014 a $2019^{* *}$.

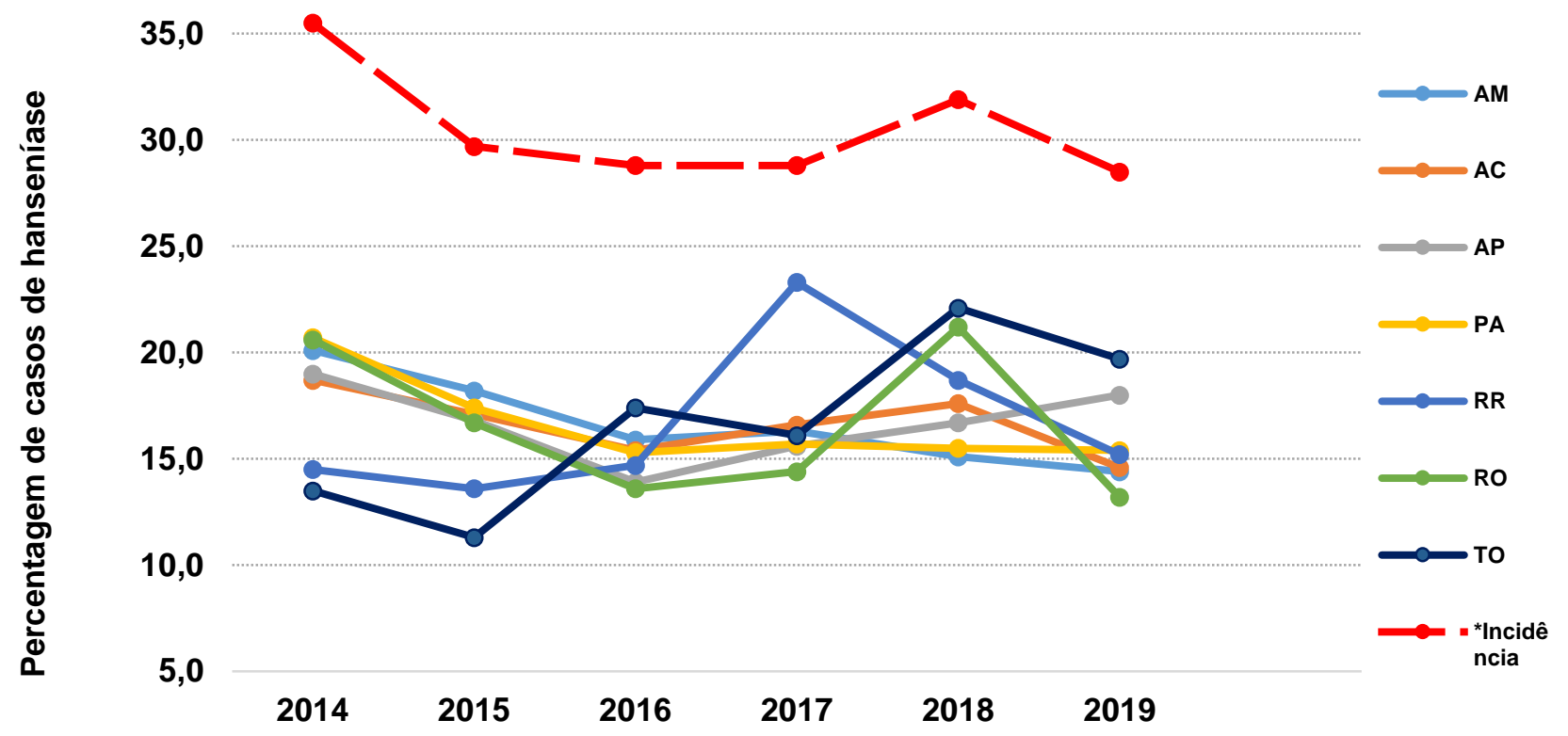

Legenda: *Taxa de Incidência anual da região Norte/100.000hab; **Dados preliminares até 05/06/2020. Sujeito a alterações.

Fonte: Oliveira DJS, et al., 2021; dados extraídos do MS/SVS/SINAN.

Embora o Brasil esteja em consonância com as recomendações estabelecidas pela Organização Mundial da Saúde (OMS), por meio da implementação da Estratégia Global para Hanseníase no período de 2016 a 2020, que visa à detecção e o tratamento imediato reduzindo a carga da doença e incapacidades físicas aos acometidos, os dados corroboram a necessidade de intensificação das ações de combate à hanseníase (OMS, 2016). Além dos objetivos estabelecidos, a OMS destaca também a responsabilidade e a relevância da coparticipação do governo com organizações no setor privado, comunitário e ONGs locais e, em nível de conhecimento especializado, considera o treinamento de trabalhadores de saúde.

Ao analisar o levantamento de Andrade RO (2019), a partir dos dados extraídos, conclui-se que o aumento de casos ocorrido em 2018 se remete a falhas na rotina de profilaxia e identificação da doença, destacando a doença como lenta e de difícil diagnóstico, portanto, concretiza-se a necessidade de profissionais capacitados no quesito diagnóstico precoce. Além disso, destaca a multirresistência a medicamento utilizado para tratamento da hanseníase, expondo o cenário de transmissão de cepas resistentes e a relevâncias de novos modelos de vigilância e intervenção em todos os níveis governamentais (ANDRADE RO, 2019; ROSA PS, et al., 2020).

Durante o período analisado, na região Norte, foi observado maior prevalência de casos no sexo masculino com $59 \%$ quando equiparado ao sexo feminino com $41 \%$ e, no quesito faixa etária, as maiores frequências concentram-se na faixa de 30 a 59 anos, somando $53,5 \%$ dos casos. Em relação à escolaridade dos indivíduos acometidos, os dados demonstram que as maiores frequência estão em indivíduos com Ensino Fundamental Incompleto com $46,2 \%$ dos casos (Tabela 1). 
Tabela 1 - Demonstra a frequência (\%) dos casos de hanseníase na região Norte, de acordo com os fatores sociodemográficos como: sexo, faixa etária e escolaridade, no período de 2014 a 2019.

\begin{tabular}{|c|c|c|c|c|c|c|c|}
\hline \multirow{2}{*}{ Sexo } & \multicolumn{7}{|c|}{$\% /$ ano } \\
\hline & 2014 & 2015 & 2016 & 2017 & 2018 & 2019 & Total \\
\hline Homens & 58,9 & 63,8 & 59,3 & 59,4 & 56,4 & 58,4 & 59,0 \\
\hline Mulheres & 41,1 & 36,2 & 40,7 & 40,6 & 43,6 & 41,6 & 41,0 \\
\hline Total & 6113 & 5181 & 5092 & 5169 & 5802 & 5261 & 32618 \\
\hline \multicolumn{8}{|l|}{ Faixa etária } \\
\hline$<9$ anos & 3,3 & 3,4 & 2,1 & 2,3 & 2,3 & 2,2 & 2,6 \\
\hline 10 a 14 anos & 7,6 & 6,9 & 6,5 & 6 & 6,1 & 5,2 & 6,4 \\
\hline 15 a 19 anos & 7,1 & 6,6 & 6,6 & 5,6 & 6,2 & 5,9 & 6,3 \\
\hline 20 a 29 anos & 14,8 & 13,4 & 13,3 & 13,6 & 12,2 & 13,4 & 13,4 \\
\hline 30 a 39 anos & 19,5 & 21,3 & 19,3 & 19,1 & 18,9 & 19,1 & 19,6 \\
\hline 40 a 49 anos & 17,6 & 16 & 17,4 & 18,5 & 19,4 & 19,5 & 18,1 \\
\hline 50 a 59 anos & 15,3 & 14,9 & 16,1 & 15,6 & 16,1 & 16 & 15,8 \\
\hline 60 a 69 anos & 9,1 & 11 & 11,3 & 12,3 & 11,6 & 11,4 & 11 \\
\hline$>70$ anos & 5,7 & 6,5 & 7,4 & 7 & 7,2 & 7,3 & 6,8 \\
\hline \multicolumn{8}{|l|}{ Escolaridade } \\
\hline Analfabeto & 8,2 & 9,3 & 8,3 & 8,6 & 7,8 & 7,6 & 8,3 \\
\hline Ensino fundamental incompleto & 49,6 & 50,5 & 48 & 45,3 & 42 & 41,5 & 46,2 \\
\hline Ensino fundamental completo & 5,1 & 4,8 & 5,4 & 5,1 & 6,6 & 6,5 & 5,6 \\
\hline Ensino médio incompleto & 7 & 5,9 & 7,1 & 7,3 & 8,2 & 6,9 & 7,1 \\
\hline Ensino médio completo & 12,5 & 12,3 & 13,4 & 13,9 & 15,6 & 16,4 & 14 \\
\hline Educação superior incompleta & 1,3 & 1,7 & 1,5 & 1,6 & 1,6 & 1,5 & 1,5 \\
\hline Educação superior completa & 2,4 & 2,2 & 3,2 & 3,3 & 3,4 & 4,8 & 3,2 \\
\hline Não se aplica & 0,8 & 0,9 & 0,6 & 0,6 & 0,6 & 0,7 & 0,7 \\
\hline Ignorado/Branco & 13,1 & 12,4 & 12,4 & 14,1 & 14,2 & 14,1 & 13,4 \\
\hline
\end{tabular}

Legenda: **Dados preliminares até 05/06/2020. Sujeito a alterações.

Fonte: Oliveira DJS, et al., 2021; dados extraídos do MS/SVS/SINAN.

O predomínio da hanseníase em homens também foi relatado no Boletim Epidemiológico do Ministério da Saúde entre os anos de 2012 a 2016 e, dados extraídos de Nobre ML, et al. (2017) e Santos MAS, et al. (2020), tal perfil reflete a maior exposição deste gênero ao Mycobacterium leprae em ambientes de risco, além de relatarem o menor cuidado com a higiene pessoal e estética, negligenciando surgimento de lesões que possam indicar uma possível patologia, portanto, impossibilitando o diagnóstico precoce. Santo MAS, et al. (2020) ainda expõe que indivíduos do sexo masculino detém maiores chances de diagnosticar incapacidade física devido, sobretudo, a baixa procura por serviços de saúde, caracterizando-os como maiores contribuintes para propagação da doença.

No que diz respeito à faixa etária predominante, é possível inferir que a população que mais sofre com a hanseníase é a economicamente ativa, corroborando sua maior exposição. $\mathrm{E}$, em uma população que contribui para massa real de rendimento ao país, a possibilidade de desenvolver lesões que provocam 
incapacidades físicas e psicológicas adjunto a gastos públicos aos serviços hospitalares, podem afetar a renda per capita e gerar um custo social elevado (LANA FCF, et al., 2004 e SOUZA EV, et al., 2020). Dessa forma, ressalta o impacto positivo dos investimentos em diagnóstico e tratamento precoce, uma vez que tais valores são, significativamente, menos onerosos.

Frequentemente a literatura expõe o grau de escolaridade como indicador de condição de vida, contudo o resultado retrata a menor escolaridade entre os acometidos, indicando a falta de informações sobre a doença e suas consequências, demonstrando a influência dos determinantes sociais no processo de adoecimento da população, interferindo diretamente no reconhecimento das manifestações clínicas da doença, na compreensão das orientações fornecidas por profissionais de saúde, e na realização do autocuidado e tratamento oportuno (MIRANZI SSC, et al., 2010; JESUS MS, et al., 2019).

Em uma análise da região Norte segundo fatores sociodemográficos, no período de 2014 a 2019 , considerando as Unidades Federativas (UF's) com maior frequência de casos, o estado do Tocantins possui a maior cobertura de Equipes de Saúde da Família (ESF), alcançando $88,2 \%$ da população, sendo possível detectar um maior número de casos novos, refletindo assim na alta taxa de incidência do estado (84\%). Diferentemente do estado do Pará que, em decorrência de sua vasta população, a incidência de casos é baixa quando comparada ao alcance da cobertura de ESF com $33,1 \%$ e $57,6 \%$, respectivamente. Podendo observar o mesmo perfil aos demais estados (Tabela 2).

De acordo com os fatores socioambientais, referente ao indicador de atendimento total de água (IN055), foram observados valores aceitáveis, variando de $34,91 \%$ a $81,14 \%$ em todos os estados, todavia, ao avaliar os indicadores de coleta de esgoto (IN015) e esgoto tratado à água consumida (IN046), os resultados expõem a baixa percentagem em $85,7 \%$ dos estados da região Norte, com mínima de 8,02\% e máxima de 33,91\%, com exceção de Roraima que concentra 70,17\% para ambos os indicadores (Tabela 2). Observa-se também que o investimento em abastecimento de água é elevado principalmente no estado do Pará, cerca de $R \$$ 51.127.869,32, em contrapartida, o investimento em esgotamento sanitário é baixo em todos estados, (exceto Roraima) (Tabela 2). 
Tabela 2 - Apresenta a percentagem e a taxa de incidência de casos, de acordo com os fatores socioambientais das Unidades Federativas da região Norte, no período de 2014 a $2019^{* *}$

\begin{tabular}{|c|c|c|c|c|c|c|c|c|c|c|}
\hline \multirow[b]{2}{*}{ Norte } & \multicolumn{10}{|c|}{ Fatores sociodemográficos/ambientais } \\
\hline & $\%$ Casos & Incidência* & Média ESF & $\begin{array}{c}\text { Cobertura } \\
\text { ESF (\%) }\end{array}$ & IDH & $\begin{array}{l}\text { IN055 } \\
(\%)\end{array}$ & $\begin{array}{l}\text { IN015 } \\
\text { (\%) }\end{array}$ & $\begin{array}{l}\text { IN046 } \\
\text { (\%) }\end{array}$ & $\begin{array}{c}\text { FN052 } \\
\text { (R\$) }\end{array}$ & $\begin{array}{l}\text { FN053 } \\
\text { (R\$) }\end{array}$ \\
\hline AM & 8,6 & 11,7 & 674 & 55,2 & 0,674 & 81,14 & 30,36 & 29,72 & - & - \\
\hline$A C$ & 2,3 & 15,1 & 206 & 68,9 & 0,663 & 47,07 & 18,78 & 18,78 & $46.404 .307,46$ & $9.976 .523,50$ \\
\hline AP & 2,0 & 13,6 & 147 & 49,7 & 0,708 & 34,91 & 15,62 & 14,8 & $4.998 .927,25$ & - \\
\hline PA & 50,8 & 33,1 & 1369 & 57,6 & 0,646 & 45,62 & 18,36 & 8,02 & $51.127 .869,32$ & $1.240 .215,64$ \\
\hline $\mathrm{RR}$ & 1,8 & 17,8 & 122 & 61,9 & 0,707 & 81,47 & 70,17 & 70,17 & - & $46.790 .664,38$ \\
\hline $\mathrm{RO}$ & 10,7 & 32,8 & 365 & 68,8 & 0,690 & 49,41 & 12,23 & 9,55 & $26.565 .555,46$ & $7.062 .492,70$ \\
\hline TO & 23,8 & 84 & 476 & 88,2 & 0,699 & 79,34 & 33,91 & 33,49 & - & - \\
\hline
\end{tabular}

Legenda: *Taxa de Incidência/100.000hab; IN055 - Índice de atendimento total de água; IN015 - Índice de coleta de esgoto; IN046 - Índice de esgoto tratado referido à água consumida; FN052 - Investimento em abastecimento de Água; FN053 - Investimento em esgotamento sanitário.

Fonte: Oliveira DJS, et al., 2021; dados extraídos do MS/SVS/SINAN (06/2020)*; SAGE; SNIS; IBGE (2019). 
Pesquisas apontam que uma maior cobertura pela Estratégia Saúde da Família no Brasil está intimamente relacionada à redução dos focos de contágio da hanseníase e contribui para o diagnóstico precoce, promovendo a adesão ao tratamento oportuno (MAGALHÃES MCC e ROJAS LI, 2007; LANZA FM, et al., 2014).

Deste modo, constata-se que a eliminação da hanseníase será alcançada somente quando as ações realizadas pelas ESF estiverem focadas na concretização dos princípios do SUS, especialmente da integralidade, tendo em vista a cronicidade da doença (MONTEIRO LD, et al., 2018).

Dados do IBGE demonstram que os estados do Norte apresenta Índice de Desenvolvimento Humano (IDH) variando de médio a alto, todavia, o IDH avalia três esferas sociais; renda, longevidade e educação, portanto, não é uma medida representativa direta das condições de saneamento básico e hábitos higiênicos. De acordo com dados extraído de Leano HAM, et al. (2019), a condição de pobreza em si não acarreta na transmissibilidade da doença, mas sim quando associada ao adensamento populacional, fatores sociodemográficos desfavoráveis e dificuldade no acesso a saneamento básico, informações e serviços de saúde, os quais são expostos na pesquisa e destacados como preocupante devido ao descaso principalmente por se tratar de fatores diretamente ligados ao ciclo de transmissão ativa da infecção e, como consequência, ocasiona a alta taxa de incidência de casos.

Em uma análise da quantidade de municípios atendidos por serviços de água e esgoto, foi observada uma totalidade das UF do Norte possui mais de $50 \%$ dos municípios com abastecimento de água, exceto Amazonas que exibe 37,1\%. Quanto ao esgotamento sanitário, 100\% dos estados apresentam-se com indicador relativamente baixo, sendo Amazonas (6,5\%), Pará (9\%) e Acre $(9,1 \%)$ com menor quantidade de municípios atendidos e, de acordo com indicador GE028, com exceção de Amazonas e Amapá, 71,4\% dos estados apresentam municípios com delegação para prestar os serviços, sendo destaque o Pará, com menor delegação $(10,4 \%)$ e com 26,4\% dos municípios com delegação vencida (Tabela 3).

Tabela 3 - Evidencia o número total de municípios e a percentagem de municípios atendidos com os indicadores de abastecimento de água e esgotamento sanitário, das Unidades Federativas da região Norte.

\begin{tabular}{ccccc}
\hline \multirow{2}{*}{ Norte } & $\mathbf{n}^{\circ}$ total municípios & \multicolumn{3}{c}{ Indicadores } \\
\cline { 3 - 5 } & 62 & $\begin{array}{c}\text { GE05a } \\
(\%)\end{array}$ & $\begin{array}{c}\text { GE05b } \\
(\%)\end{array}$ & $\begin{array}{c}\text { GE028 } \\
(\%)\end{array}$ \\
\hline AM & 37,1 & 6,5 & - \\
AC & 22 & 100 & 9,1 & 90,9 \\
AP & 16 & 100 & 37,5 & - \\
PA & 144 & 59,7 & 9 & $10,4^{*}$ \\
RR & 15 & 100 & 26,7 & 73,3 \\
RO & 52 & 90,4 & 13,5 & 36,5 \\
TO & 140 & 100 & 13,6 & 66,3 \\
\hline
\end{tabular}

Legenda: GE05a - Quantidade total de municípios atendidos com abastecimento de água; GE05b Quantidade total de municípios atendidos com esgotamento sanitário; GE028 - Quantidade de municípios não atendidos com esgotamento sanitário, mas com delegação em vigor para prestar esses serviços; *38 (26,4\%) municípios com delegação vencida.

Fonte: Oliveira DJS, et al., 2021; dados extraídos do SNIS, 2018.

Tendo em vista que as condições de moradia inadequadas associadas à falta de saneamento básico são fatores determinantes à promoção de saúde, estes aspectos implicam negativamente no controle da doença (MONTEIRO LD, et al., 2017). Além da hanseníase, a falta de acesso aos serviços básico de saneamento contribui negativamente à saúde humana, acarretando as chamadas Doenças Relacionadas ao Saneamento Ambiental Inadequado (DRSAI) que, segundo o Instituto Trata Brasil, foram responsáveis por cerca de 12,46 internações por 10 mil habitantes no ano de 2017 (SIQUEIRA MS, et al., 2017; TRATA BRASIL, 2019). A 
precariedade dos serviços adjunta aos baixos investimentos em esgotamento sanitário gera elevado gastos públicos e privados com tratamento de doenças.

Segundo Reis DA, et al. (2017), é relevante destacar o papel crucial do Estado no quesito qualidade de serviços, além da atuação ativa dos municípios quanto a elaboração dos Planos Municipais de Saneamento Básico (PMSB) e a prestação dos serviços.

A partir de uma análise isolada dos municípios do estado do Pará que possuem mais de 100.000 habitantes, no período de 2014 a 2019, foi observado 18 municípios com elevada taxa de incidência de casos de hanseníase com mínima de 15,1 e máxima de 64,2. Considerando as três cidades com maiores taxas de incidência, destaca-se Tailândia (64,2\%), Altamira (61,6\%) e Parauapebas (60\%), sendo Tailandia responsável pela menor cobertura de equipes de Saúde da Família $(28,4 \%)$; e com menor incidência, Santarém, Bragança e Belém com 15,1\%; 15,9\% e 17,6\%, respectivamente (Tabela 4).

Apesar das equipes Saúde da Família alcançarem uma grande cobertura populacional, em média 3.000 indivíduos, e serem capazes de detectar o maior número de casos, Belém não se configura neste aspecto devido seu amplo território, abrangendo apenas $21,2 \%$ da cobertura ESF (Tabela 4).

Tabela 4 - Demonstra a percentagem e incidência de casos de hanseníase segundo fatores socioambientais, dos munícipios do Estado do Pará que apresentam mais de 100.000hab, no período de 2014 a 2019***.

\begin{tabular}{cccccc}
\hline & & & \multicolumn{2}{c}{ Fatores socioambientais } \\
\cline { 5 - 6 } Municípios PA* & \% Casos & $\begin{array}{c}\text { Taxa de } \\
\text { Incidência** }\end{array}$ & IDHM & $\mathbf{n}^{\circ}$ de ESF & $\begin{array}{c}\text { Cobertura ESF } \\
\text { (\%) }\end{array}$ \\
\hline Bragança & 1,6 & 15,9 & 0.600 & 49 & 131,1 \\
Barcarena & 1,9 & 19,8 & 0.662 & 31 & 84,1 \\
Cametá & 2,8 & 26,3 & 0.577 & 18 & 44,5 \\
Breves & 3,0 & 37 & 0.503 & 15 & 50 \\
Marituba & 3,2 & 31,8 & 0.676 & 21 & 54,1 \\
Itaituba & 3,2 & 39,7 & 0.640 & 21 & 71,4 \\
Abaetetuba & 3,3 & 27,3 & 0.628 & 19 & 41,2 \\
Santarém & 3,6 & 15,1 & 0.691 & 36 & 40,5 \\
Tucuruí & 3,7 & 42,1 & 0.666 & 23 & 68,9 \\
Castanhal & 3,9 & 25,1 & 0.673 & 49 & 83,1 \\
São Félix do Xingu & 3,9 & 40,2 & 0,594 & 17 & 44,3 \\
Paragominas & 5,1 & 58,8 & 0.645 & 23 & 69,2 \\
Tailândia & 5,2 & 64,2 & 0.588 & 9 & 28,4 \\
Altamira & 5,5 & 61,6 & 0.665 & 22 & 65,4 \\
Ananindeua & 8,6 & 20,8 & 0.718 & 115 & 74,1 \\
Parauapebas & 9,5 & 60 & 0.715 & 34 & 54,9 \\
Marabá & 11,7 & 54,2 & 0.668 & 20 & 24,3 \\
Belém & 20,5 & 17,6 & 0.746 & 95 & 21,2 \\
\hline
\end{tabular}

Legenda: *Municípios com mais de 100.000hab; **Taxa de Incidência anual da região Norte/100.000hab; ${ }^{* * *}$ Dados preliminares até $05 / 06 / 2020$. Sujeito a alterações.

Fonte: Oliveira DJS, et al., 2021; dados extraídos do MS/SVS/SINAN.

O munícipio de Belém é caracterizado como de alta carga da doença, com 20,5\% dos casos, pode-se relacionar esse fato ao elevado fluxo migratório provocado pela região economicamente mais desenvolvida do Pará, no entanto a cobertura por ESF é baixa e não alcança a totalidade da população, devido ao seu amplo território (CHAVES EC, et al., 2017). 
Ademais, sabe-se que a presença de uma equipe multiprofissional é de extrema relevância, tendo em vista a necessidade do fornecimento de cuidados de maneira integral a esses usuários, promovendo um atendimento acolhedor, uma maior resolutividade do trabalho e consequentemente uma melhor assistência da atenção primária. Deste modo, a baixa capacitação de profissionais também têm influência na baixa cobertura de ESF em alguns municípios, visto que, os treinamentos geralmente são voltados para profissionais de nível superior, não atendendo à demandas de profissionais como agentes comunitários de saúde ou técnico/auxiliar de enfermagem (SOUSA GS, et al., 2017).

Segundo o Conselho Regional de Medicina do Estado do Pará (2017), Tailândia se encontra em sexta posição no ranking dos 20 municípios com menor gasto per capita em ações e serviços públicos em saúde, sendo um valor equivalente a $\mathrm{R} \$ 84,43$ por habitante, este dado representa o baixo investimento em saúde, que, por sua vez, reflete na baixa cobertura de ESF e alta taxa de incidência de casos, influenciando na qualidade de vida da população e na assistência em saúde.

Diante disto, as atividades dos programas de controle precisam ser focadas em estados e municípios com maior vulnerabilidade social, por meio de investimentos direcionados para a melhoria das condições de vida populacionais. A boa cobertura pelas ESF, com a composição de uma equipe multiprofissional capacitada, assegura a descoberta dos casos, mas não é suficiente para o efetivo controle, uma vez que se deve ter uma visão abrangente também para os determinantes sociais que desencadeiam a doença (CAVALCANTE MDMA, 2018 e PEREIRA WMM, et al., 2019).

\section{CONCLUSÃO}

Com a análise do perfil epidemiológico da Hanseníase e a distribuição espacial e demográfica na região Norte, segundo o período de estudo, pode-se concluir que a taxa de incidência demonstrou relação com a cobertura das ESF e os fatores sociodemográficos/ambientais, o que incita a um diagnóstico precoce. Portanto, enfatiza-se a relevância do desenvolvimento de ações públicas e educativas para a comunidade, no que se refere à divulgação de informações sobre a hanseníase e suas particularidades, para a busca ativa dos casos e maior procura desta população aos serviços de atenção básica de saúde, gerando o aumento de diagnósticos precoces, além de um tratamento adequado da doença e redução dos graus de incapacidade dos pacientes acometidos.

\section{REFERÊNCIAS}

1. ANDRADE RO. A persistência da hanseníase. Revista Pesquisa Fapesp. Set. 2019.

2. BASEGGIO RC. Determinantes Sociais e a Hanseníase na População Feminina no Estado do Paraná. Dissertação de Mestrado (Programa De Mestrado Em Biociências E Saúde). Universidade do Oeste de Santa Catarina UNOESC. Joaçaba. 2016.

3. BRASIL. Ministério da Saúde. Secretaria de Políticas de Saúde. Departamento de Atenção Básica. Controle da hanseníase na atenção básica: guia prático para profissionais da equipe de saúde da família. Série A. Normas e Manuais técnicos. Brasília, 2001; 111.

4. BRASIL. Secretaria de Vigilância em Saúde. Ministério da Saúde. Boletim Epidemiológico - Caracterização da situação epidemiológica da hanseníase e diferenças por sexo, Brasil, 2012-2016. 2018; 49(4).

5. BRASIL. Secretaria de Vigilância em Saúde. Ministério da Saúde. Boletim Epidemiológico. Número Especial, 2020.

6. CAVALCANTE MDMA. Gestão do cuidado à Hanseníase: Estudo de caso em um serviço de referência na $5^{\mathrm{a}}$ Regional de Saúde, Paraná, Brasil. Tese (Doutorado) - Programa de Pós-Graduação em Enfermagem. Setor de Ciências da Saúde. Universidade Federal do Paraná, Curitiba, 2018.

7. CAVALCANTI CDTV, SILVA CGC. Situação de Hanseníase no Município de Cabrobó, Pe, 2001 a 2007. Monografia (Especialização em Gestão de Sistemas e Serviços de Saúde) - Departamento de Saúde Coletiva. Centro Pesquisa Aggeu Magalhães. Fundação Oswaldo Cruz. Recife, 2008; 28.

8. CHAVES EC, et al. Índice de carência social e hanseníase no estado do Pará em 2013: análise espacial. Revista Epidemiologia e Serviços de Saúde, Brasília, 2017; 26(4): 807-816.

9. CONSELHO REGIONAL DE MEDICINA DO ESTADO DO PARÁ. Cametá, Bragança e Ananindeua figuram no topo nacional do baixo investimento em saúde, 2017.

10. GONÇALVES NV, et al. A hanseníase em um distrito administrativo de Belém, estado do Pará, Brasil: relações entre território, socioeconomia e política pública em saúde, 2007-2013. Revista Pan-Amazônica Saúde, 2018; 9(2). 
11. JESUS MS, et al. Características epidemiológicas e análise espacial dos casos de hanseníase em um município endêmico. Rev Rene, 2019; 20.

12. JUNIOR FACP. Motivos do Abandono ou Interrupção do Tratamento da Hanseníase: Uma Revisão Sistemática Da Literatura. Artigo científico (Especialização em Saúde Coletiva) - Centro de Pesquisa Aggeu Magalhães. Fundação Oswaldo Cruz. Recife, 2010.

13. LANA FCF, et al. Estimativa da prevalência oculta da hanseníase no Vale do Jequitinhonha: Minas Gerais. Rev Min Enferm, 2004; 8: 295-300.

14. LANZA FM, et al. Avaliação da Atenção Primária no controle da hanseníase: proposta de uma ferramenta destinada aos usuários. Rev Esc Enferm USP, 2014; 48(6): 1054-61.

15. MAGALHÃES MCC, ROJAS LI. Diferenciação territorial da hanseníase no Brasil. Epidemiologia e Serviços de Saúde, 2007; 16(2): 75-84.

16. MENDONÇA VA, et al. Imunologia da Hanseníase. Anais Brasileiros de Dermatologia. Rio de Janeiro, 2008;8(4).

17. MIRANZI SSC, et al. Perfil epidemiológico da hanseníase em um município brasileiro, no período de 2000 a 2006. Revista da Sociedade Brasileira de Medicina Tropical, 2010; 43(1): 62-67.

18. MONTEIRO LD, et al. Determinantes sociais da hanseníase em um estado hiperendêmico da região Norte do Brasil. Rev Saude Publica, 2017; 51:70.

19. MONTEIRO LD, et al. Tendências da hanseníase após implementação de um projeto de intervenção em uma capital da Região Norte do Brasil, 2002-2016. Cadernos de Saúde Pública, 2018.

20. MOURA ADA, et al. Perfil dos portadores de hanseníase de um centro de referência de um estado brasileiro. Rev enferm UERJ, 2016; 24(6): 9625.

21. NASCIMENTO DS, et al. Limitação de atividade e restrição à participação social em pessoas com hanseníase: análise transversal da magnitude e fatores associados em município hiperendêmico do Piauí, 2001 a 2014. Revista Epidemiologia e Serviços de Saúde, 2020; 29(3).

22. NOBRE ML, et al. Multibacillary leprosy by population groups in Brazil: Lessons from an observational study. Plos Neglected Tropical Diseases, 2017; 11(2).

23. PEREIRA WMM, et al. Hanseníase em metrópole da Amazônia brasileira: cobertura de atenção básica à saúde e sua relação com o perfil clínico e a distribuição espaço-temporal da doença em Belém, estado do Pará, Brasil, de 2006 a 2015. Rev Pan Amaz Saude, 2019; 2176-6223.

24. RIBEIRO MDA, et al. Estudo epidemiológico da hanseníase no Brasil: reflexão sobre as metas de eliminação. Revista Panamericana de Salud Pública, 2018; $42: 42$.

25. ROSA PS, et al. Emergence and transmission of drug/multidrug-resistant Mycobacterium leprae in a former leprosy colony in the Brazilian Amazon. Clinical Infectious Diseases, 2019; 6(10):2054-2061.

26. SANTOS KCB, et al. Estratégias de controle e vigilância de contatos de hanseníase: revisão integrativa. Revista Saúde Debate, 2019; 43(121): 576-591.

27. SANTOS MAS, et al. Análise dos aspectos clínicos de pacientes com hanseníase entre 2010-2017 em um centro de referência em Campina. An Bras Dermatol, 2020; 95 (2): 252-254.

28. SIQUEIRA MS, et al. Internações por doenças relacionadas ao saneamento ambiental inadequado na rede pública de saúde da região metropolitana de Porto Alegre, Rio Grande do Sul, 2010-2014. Epidemiol. Serv. Saude, Brasília, 2017; 26(4): 795-806.

29. SILVA AR, et al. Hanseníase no Município de Buriticupu, Estado do Maranhão: busca ativa de casos na população adulta. Revista da Sociedade Brasileira de Medicina Tropical, Uberaba, 2010; 43(6): 691-694.

30. SOUSA GS, et al. Hanseníase e Atenção Primária à Saúde: uma avaliação de estrutura do programa. Saúde Debate, 2017; 41(112): 230-242.

31. SOUZA CDF, et al. Modelagem espacial da hanseníase no estado da Bahia, Brasil, (2001 - 2015) e determinantes sociais da saúde. Revista Epidemiologia e Serviços de Saúde, Brasília, 2020; 28(1).

32. SOUZA EV, et al. Hanseníase: epidemiologia da morbimortalidade e gastos públicos no nordeste brasileiro, 2020; 12:1150-1156.

33. TRATA BRASIL. Internações de doenças por veiculação hídrica no Brasil, 2019.

34. WORLD HEALTH ORGANIZATION (WHO). Global Leprosy Strategy: Accelerating towards a leprosy-free world. WHO, 2016. 